\title{
ROLE OF TUMOR NECROSIS FACTOR ALPHA AND BASIC FIBROBLAST GROWTH FACTOR IN THE PATHOGENESIS OF VITILIGO:AN IMMUNOHISTOCHEMICAL STUDY BEFORE AND AFTER MELANOCYTE TRANSFER BY SANDPAPER TECHNIQUE

\author{
Nagwa Ali Diab,Magda Ibrahim Assaf, Mohamed Abdelkader Toama, and Mohamed Ibrahim \\ Elghareeb \\ Departments of Dermatology and Pathology.Faculty of medicine,Zagazig university.
}

\begin{abstract}
Background: Vitiligo is an acquired depigmentation of skin and mucous membranes. Many theories explain its pathogenesis. Keratinocyte dysfunction in vitiligo may be a possible link between the various pathological changes occurring in vitiliginous skin. Keratinocytes produce several mediators, cytokines and growth factors that support or inhibit the growth and/or melanization of surrounding melanocytes. Basic fibroblastic growth factor (bFGF) is proposed to be a paracrine mitogen for human melanocytes.Tumor necrosis factor $\alpha(\mathrm{TNF} \alpha)$, is suggested to be as an early paracrine inhibitors of melanocyte.In this study,we evaluated the keratinocyte 's TNF $\alpha$ and b-FGF in vitiligo patients before and after treatment with melanocyte transfer by sandpaper technique.
\end{abstract}

Methods: This study was carried out on two groups, 17 vitiligo patients , and 17 healthy persons as control. Skin biopsies were taken from the patients before and after treatment with melanocyte transfer from the lesional and perilesional skin .Skin biopsies were taken also from skin of the normal control individuals. These biopsies were prepared for histological evaluation and immunohistochemical(IHC) staining forTNF $\alpha$ b-FGF.Evaluation of IHC slides was done by a subjective method(i.e. eyes of an expert pathologist) and an objective method(Image analyser) before and after 3 months of treatment with melanocyte transfer by sandpaper technique.

Results: There was a statistically highly significant increase of b-FGF staining in lesional and perilesional skin of the patient's group in comparison with their staining in normal control. Regarding TNF $\alpha$ staining of lesional and perilesional skin,there were a statistically significant increase and a statistically highly significant increase respectively with its staining in the normal control. The clinical results of the melanocyte transfer by sandpaper technique, after 3 months of follow up, were as follow,47.1\%of patients showed mild improvement,35.3\% of patients showed good improvement,and $17.6 \%$ of patients showed no improvement.TNF $\alpha$ staining of lesional and perilesional skin,evaluated subjectively, showed a statistically highly significant increase after treatment with melanocyte transfer in comparison with their staining before treatment.Regarding b-FGF staining, subjectively evaluated,there was a statistically highly significant increase after treatment in lesional skin and a statistically highly significant decrease after treatment in perilesional skin.Using image analyser as an objective method of evaluation,there was a statistically significant increase of $\mathrm{TNF} \alpha$ staining of lesional and perilesional skin after melanocyte transfer in comparison with their staining before treatment.On comparing b-FGF stainings after treatment with their corresponders before treatment by image analyser,there was a statistically significant decrease of b-FGF staining in lesional skin after treatment and there was no significant change in perilesional skin before and after treatment.

Conclusion:change in the microenvironment of vitiliginous skin is evident .These changes in the cytokines,growth factors affecting the melanocytes may be a joint step in the pathogenesis of vitiligo between an intial step (supposed to be oxidative stress and apoptosis in keratinocytes) and an eventual step marked by destruction of the melanocytes.

Key Words:Vitiligo,IHC,b-FGF,TNF $\alpha$,melanocytes,and Image analyser

Corresponding author: Mohamed Ibrahim Elghareeb

Received: 13 September 2015 tel:01092907455

Accepted: 30 September 2015

E.mail:elghareeb.mohamed@yahoo.com

\section{INTRODUCTION}

V itiligo is an acquired chronic disorder of the skin and mucous membranes that is characterized by well circumscribed, depigmented macules and patches and that occurs secondary to selective destruction of melanocytes. It may appear at any age.Some cases have been reported as early as 6 weeks after birth ${ }^{[1]}$.

The cause of vitiligo is unknown. There are many pathophysiologic theories; the most prominent are autoimmune, neurohumoral, 
autocytotoxic, and genetic. None are exclusive, as it seems that each partially contributes in the pathogenesis of vitiligo ${ }^{[2]}$. Recently, Keratinocyte dysfunction in vitiligo appeared as a new era in the pathogenesis of vitiligo. Keratinocytes produce several mediators, cytokines and growth factors that support or inhibit the growth and/or melanization of surrounding melanocytes. Basic fibroblastic growth factor (bFGF) is proposed to be a paracrine mitogen for human melanocytes. Tumor necrosis factor $\alpha(\mathrm{TNF} \alpha)$, is suggested to be as an early paracrine inhibitors of melanocytes ${ }^{[3]}$.

Changes of the levels of these keratinocytederived molecules are described in vitiligo epidermis, suggesting a role of epidermal cytokines in the pathogenesis of vitiligo ${ }^{[4]}$.

\section{PATIENTS AND METHODS}

This study was carried out on two groups, 17 vitiligo patients, who represented patients group, and 17 healthy persons, who represented control group.

Every patient was subjected to the following:

I-Thorough history taking

II-Examination: documenting the

distribution, anatomical site and extent of the vitiliginous lesions.

III-Photographs:All patients were photographed before and after treatment

$\boldsymbol{I V}$-Written and informed consents were obtained from all subjects.

\section{V-Skin biopspies before treatment:}

A $5 \mathrm{~mm}$ punch biopsy was taken from lesional skin and $3 \mathrm{~mm}$ punch biopsy from the surrounding normal skin of every patient.A 5 $\mathrm{mm}$ punch biopsy was taken also from the skin of the normal control individuals.

Each specimen was fixed in $10 \%$ neutral formalin solution and embedded in paraffin to form paraffin blocks .

Serial sections of $5 \mu$ thickness were prepared from each paraffin block,one of them stained by Haematoxylin and $\operatorname{eosin}(\mathrm{H} \& \mathrm{E})$ for histological evaluation,2 other sections were mounted on charged slides for immunohistochemical staining (IHC)using the following:

A- Primary antibodies:

Tumor necrosis factor alpha (TNF $\alpha)$ antibody(TNFa (52B83): sc-52746, SANTA CRUZ BIOTECHNOLOGY®, INC. with a dilution of 1:50) and basic Fibroblast growth factor (b-FGF) antibody(FGF-2 (147): sc-79, SANTA CRUZ BIOTECHNOLOGY®, INC. with a dilution of 1:50).

B- Universal kit:

An improved Biotin-Streptvidin amplified(B-SA) detection system was used to detect specific antigens in formalin-fixed, paraffin-embedded tissue. The procedure of immunohistochemical staining followed the guidelines of Shi et al, $1995^{[5]}$.

Evaluation of the immunohistochemical staining results :by

1) Detection of the percentage of nuclear staining of keratinocytes through the whole epidermis at high magnification $(\times 400)$

2) Detection of percentage of nuclear staining of keratinocytes through the whole epidermis by the use of Image Analyser(Immuno Ratioß)after scanning the microscopic slides and converting them to digital images.

We measured mainly the keratinocyte nuclear staining of both TNF $\alpha$ and b-FGF before and after treatment to follow up the keratinocytes synthesis of these cytokines rather than their response themselves to these cytokines.

The percentage range of keratinocyte nuclear staining of TNF $\alpha$ and b-FGF in the normal control individuals was used as a reference value.Any value below the lower limit of this range was considered negative.

\section{Image Analyser(Immuno Ratio®):}

It is a software that offers a quantitative information about acquired images from IHC slides.

Image analyser can detect the site of staining(nuclear or membranous)and how much the staining there ${ }^{[6]}$.

VI-Autologous melanocyte transfer was done to all patients by Sandpaper technique $^{[7]}$ :

The skin of the donor area (normally pigmented)was manually abraded by a watersterilized $5 \times 5 \mathrm{~cm}$ piece of sandpaper until the pinpoint bleeding appears.

Dermabrasion by another piece of sandpaper was performed in the recipient area(vitiliginous area) starting from the peripheral edge toward the center of the lesion.Dermabrasion was done until the pinpoint bleeding appears . 
The sandpaper with the epidermal fragments from the donor area was applied to the dermabraded recipient area. A waterproof dressing was applied to keep the sandpaper firmly attached to the recipient area.

The donor area was cleansed with saline and covered with paraffin gauze, in order to heal by secondary intention.

Dressings of the recipient and donor areas were left in place for 7 to 10 days before being removed.

After removing the dressings, the patients had exposed to direct sunlight for 5 minutes daily.

Digital photographs were taken before melanocyte transfer to record the baseline. After the procedure, serial photographs were taken monthly until the third month of followup.

Repigmentation of receipt area was evaluated 10 days, 1,2 , and 3 months after the transfer.

Pigmentation quality was assessed using the following scale ${ }^{[7]}$ :

(0) no response or lack of pigment, $(+)$ erythema in the treated area,

$(++)$ pigmentation lighter than the normal skin,

$(+++)$ pigmentation similar to the normal skin,

$(++++)$ pigmentation darker than the normal skin.

The grade $(0)$ was considered as no improvement, while $(+),(++)$ grades as mild improvement, and $(+++),(++++)$ grades as good improvement

VII- Tumor necrosis factor-alpha and
bFGF immunohistochemically from the treated and the surrounding area- after the 3 months of treatment evaluated both subjectively and objectively using the image analyser.

\section{RESULTS}

Statistical presentation and analysis of the present study was conducted, using the mean,standard deviation, Analysis of variance(ANOVA) test,chi square test,paired t test and Mann-Whitney U test(MW) test by SPSS V.22 and when appropriate.

The results of immunohistochemical staining of normal control individuals revealed that the percentage of keratinocyte nuclear staining of TNFa ranged from 0 to $6 \%$ and that of b-FGF was from 12 to $25 \%$.

The results of the subjective evaluation method of the immunohistochemical slides of the lesional and perilesional skin of patient's group and the normal healthy skin of the control group were compared. There was a statistically highly significant increase of keratinocyte nuclear staining of b-FGF in lesional and perilesional skin in comparison with their staining in normal control. Regarding the keratinocyte nuclear staining of $\mathrm{TNF} \alpha$ of lesional and perilesional skin,there were a statistically significant increase and a statistically highly significant increase respectively with its keratinocyte nuclear staining in the normal control.

There were no significant difference between TNFa and b-FGF staining of lesional and perilesional skin in the patient's group before treatment.

The clinical results of the melanocyte keratinocyte comixture transfer by sandpaper technique were as follow, $47.1 \%$ of patients showed mild improvement,35.3\% of patients showed good improvement,and $17.6 \%$ of patients showed no improvement.

The results of the subjective evaluation method of the lesional and perilesional skin after treatment regarding $\mathrm{TNF} \alpha$ keratinocyte nuclear staining were compared with their corresponders before treatment.The nuclear staining of TNF $\alpha$ of lesional and perilesional skin showed a statistically highly significant increase after treatment with melanocyte transfer in comparison with their staining before treatment(Tab 1). 
Table(1):Lesional and perilesional keratinocytes

nuclear

TNF- $\alpha$

staining immunohistochemically evaluated by the subjective method before and after melanocyte transfer:

\begin{tabular}{ccccc}
\hline & TNF(A) & TNF(A') & Paired t & p \\
\hline Percentage(\%): & & & & \\
Range & $0-60$ & $2.47-70$ & 3.28 & $<0.001^{* *}$ \\
$\overline{\mathrm{X}} \pm$ SD & $8.6 \pm 14.7$ & $23.5 \pm 20$ & & \\
Median & 8.57 & 20 & & \\
\hline & TNF(B) & TNF(B') & Paired t & P \\
\hline Percentage(\%): & $0-50$ & & & \\
Range & $17.1 \pm 11.9$ & $1.22-65$ & 5.6 & $<0.001^{* *}$ \\
$\bar{X} \pm$ SD & 18.12 & $25.8 \pm 19$ & & \\
Median & 20 & & \\
\hline
\end{tabular}

TNF(A)=Tumor necrosis factor $\alpha$ in lesional skin before treatment, $\mathbf{T N F}\left(\mathbf{A}^{\prime}\right)=$ Tumor necrosis factor $\alpha$ in lesional skin after treatment, $\mathbf{T N F}(\mathbf{B})=$ Tumor necrosis factor $\alpha$ in perilesional skin before treatment, $\mathbf{T N F}\left(\mathbf{B}^{\prime}\right)=$ Tumor necrosis factor $\alpha$ in perilesional skin after treatment $\mathbf{N}=$ number, and $\%=$ percentage.

The results of the subjective evaluation method of the lesional and perilesional skin

Table(2):Lesional and perilesional keratinocytes nuclear b-FGF staining immunohistochemically by the subjective method before and after melanocyte transfer:

\begin{tabular}{ccccc}
\hline & FGF(A) & FGF(A') & Paired t & p \\
\hline Percentage(\%): & & & & \\
Range & $10-86.7$ & $1.13-90$ & 8.56 & $<0.001^{* *}$ \\
$\overline{\mathrm{X}} \pm$ SD & $32 \pm 22$ & $38.9 \pm 21$ & & \\
Median & 47.5 & 35 & & $\mathbf{p}$ \\
\hline & FGF(B) & FGF(B') & Paired t & \\
\hline Percentage(\%): & $3-70$ & & & \\
Range & $38.2 \pm 19.4$ & $35.5 \pm 15.7$ & & $<0.001^{* *}$ \\
$\overline{\mathrm{X}} \pm$ SD & 41.1 & 35 & & \\
Median & & &
\end{tabular}

FGF(A)=basic-fibroblast growth factor in lesional skin before treatment, $\mathbf{F G F}\left(\mathbf{A}^{\top}\right)=$ basic-fibroblast growth factor in lesional skin after treatment, $\mathbf{F G F}(\mathbf{B})=$ basic-fibroblast growth factor in perilesional skin before treatment, $\mathbf{F G F}\left(\mathbf{B}^{\prime}\right)=$ basic-fibroblast growth factor in perilesional skin after treatment, $\mathbf{N}=$ number, and $\%=$ percentage

Comparing the staining results of TNF $\alpha$ and b-FGF in lesional and perilesional skin after treatment was evaluated and showed no significant difference

The results of the objective evaluation method of the lesional and perilesional skin after treatment regarding $\mathrm{TNF} \alpha$ keratinocyte nuclear staining were compared with their corresponders before treatment.

There was a statistically significant increase of nuclear staining of TNFa of lesional and perilesional skin after melanocyte transfer,using the image analyser(Tab3). 
Table( 3 ): Lesional and perilesional keratinocytes nuclear TNF- $\alpha$ staining immunohistochemically evaluated by the objective method by Image Analyser(Immunoratio $\left.{ }^{\circledR}\right)$ before and after melanocyte transfer:

\begin{tabular}{cccccc}
\hline & Range & $\overline{\mathbf{X}} \pm$ SD & Median & Paired t & p \\
\cline { 1 - 4 } TNF(A) & $8.87-88.6$ & $36.2 \pm 24.3$ & 29 & 2.1 & $<0.05^{*}$ \\
\cline { 1 - 4 } TNF(A $)$ & $5.57-98.45$ & $42.9 \pm 27.1$ & 36 & & $<0.05^{*}$ \\
\hline TNF(B) & $9.3-99.6$ & $27.3 \pm 25.6$ & 17.95 & 2.08 & \\
\hline TNF(B ${ }^{\prime}$ ) & $13.8-98.03$ & $50.7 \pm 22.9$ & 53.07 & & \\
\hline
\end{tabular}

TNF(A)=Tumor necrosis factor $\alpha$ in lesional skin before treatment, $\mathbf{T N F}\left(\mathbf{A}^{\prime}\right)=$ Tumor necrosis factor $\alpha$ in lesional skin after treatment, $\mathbf{T N F}(\mathbf{B})=$ Tumor necrosis factor $\alpha$ in perilesional skin before treatment, $\mathbf{T N F}\left(\mathbf{B}^{\prime}\right)=$ Tumor necrosis factor $\alpha$ in perilesional skin after treatment, and $\overline{\mathbf{X}} \pm \mathbf{S D}=$ Mean \pm Standard deviation

The results of the objective evaluation method of the lesional and perilesional skin

Table(4): Lesional and perilesional keratinocytes nuclear b-FGF staining immunohistochemically evaluated by the objective method by Image Analyser(Immunoratio $\left.{ }^{\circledR}\right)$ before and after melanocyte transfer:

\begin{tabular}{cccccc}
\hline & Range & $\overline{\mathbf{X}} \pm$ SD & Median & Paired t & p \\
\cline { 1 - 4 } FGF(A) & $48.37-100$ & $91.8 \pm 15.1$ & 100 & 2.06 & $<0.05^{*}$ \\
\cline { 1 - 4 } FGF(A) & $15.37-100$ & $81.4 \pm 22.6$ & 88.35 & & \\
\hline FGF(B) & $12.1-100$ & $86.1 \pm 26.6$ & 100 & 0.95 & $>0.05(\mathrm{NS})$ \\
\hline FGF(B) & $10-99.8$ & $77.1 \pm 26.8$ & 90.15 & & \\
\hline
\end{tabular}

FGF(A)=basic-fibroblast growth factor in lesional skin before treatment, $\mathbf{F G F}\left(\mathbf{A}^{\prime}\right)=$ basic-fibroblast growth factor in lesional skin after treatment, $\mathbf{F G F}(\mathbf{B})=$ basic-fibroblast growth factor in perilesional skin before treatment, $\mathbf{F G F}\left(\mathbf{B}^{`}\right)=$ basic-fibroblast growth factor in perilesional skin after treatment, and $\overline{\mathbf{X}} \pm \mathbf{S D}=$ Mean \pm Standard deviation

Correlation between the results of both evaluation methods (i.e. subjective and objective)was done. There was a significant correlation between both methods in keratinocyte nuclear staining of $\mathrm{TNF} \alpha$ in lesional and perilesional skin before treatment with melanocyte transfer.

There was a highly significant correlation and a significant correlation between the two methods in nuclear staining of $\mathrm{TNF} \alpha$ in lesional and perilesional skin respectively after treatment with melanocyte transfer.

There was no significant correlation between both methods in the keratinocyte nuclear staining of b-FGF in lesional and perilesional skin before and after treatment with melanocyte transfer. 


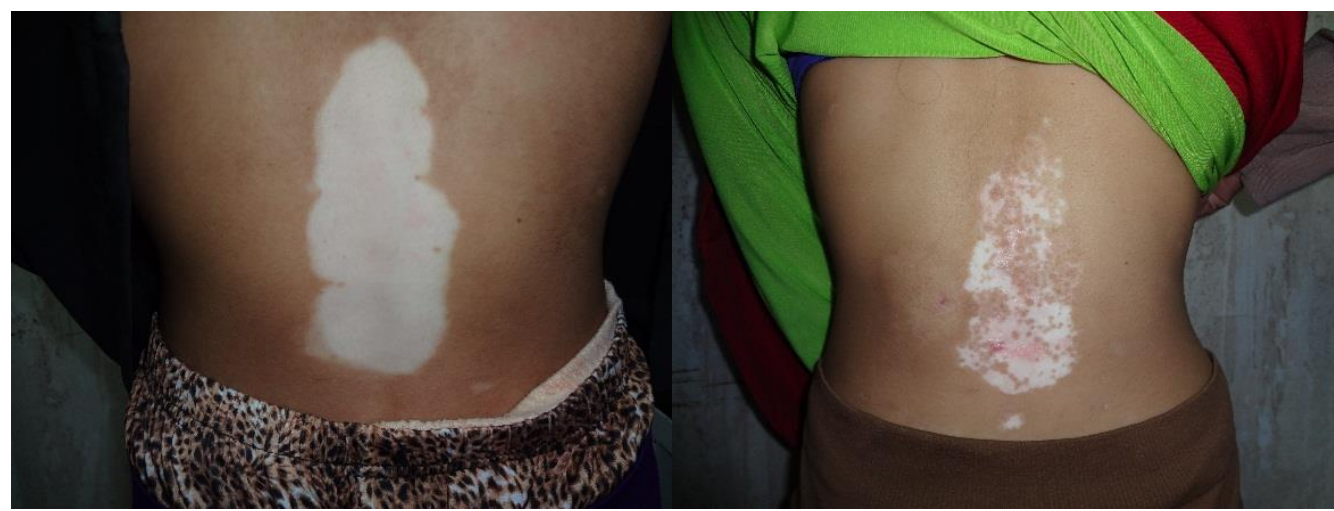

(a)

(b)

Fig(1): vitiliginous patch in the sacral area before(a) and after 3 months(b)of melanocyte transfer by sandpaper technique( showing good improvement).

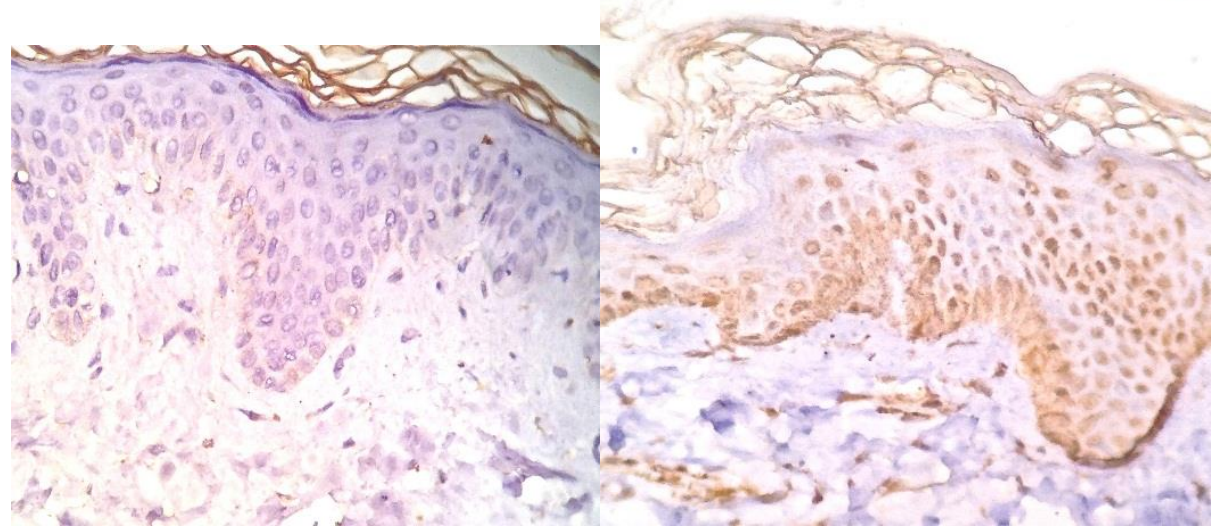

(a)

(b)

Fig(2): IHC staining of TNFa of lesional skin before(a) and after(b) 3 months of melanocyte transfer by sandpaper technique(TNF $\alpha$ staining $\times 400)$.

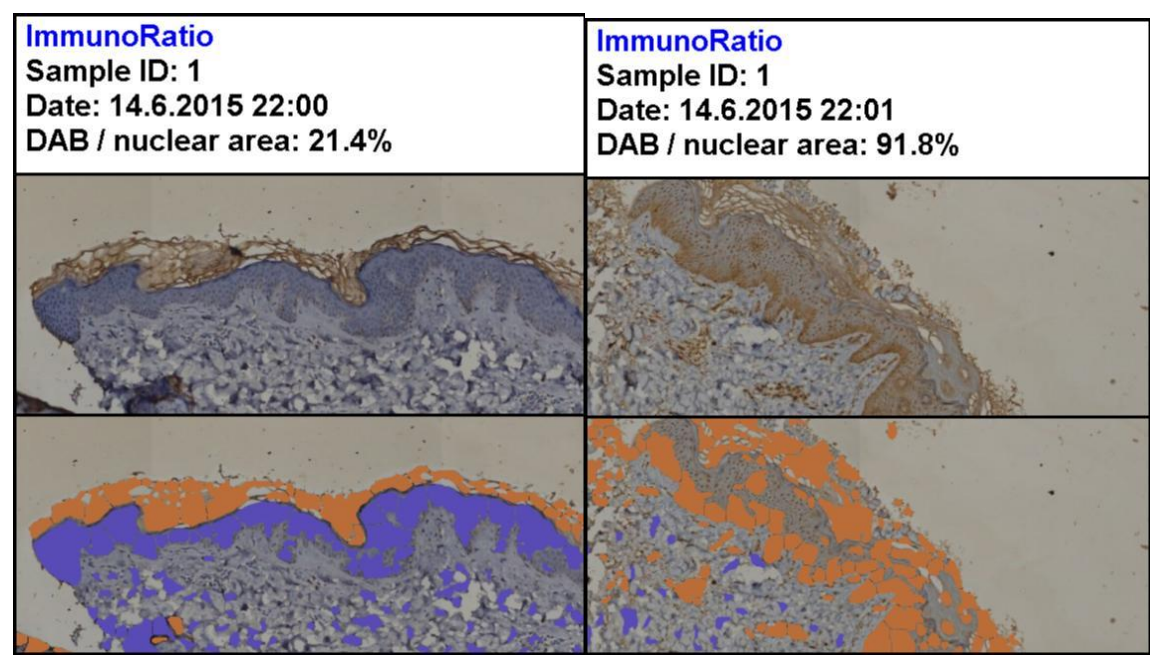

(a)

(b)

Fig (3): Image analyser of TNF $\alpha$ staining of lesional skin before(a) and after(b) 3 months of melanocyte transfer by sandpaper technique. 


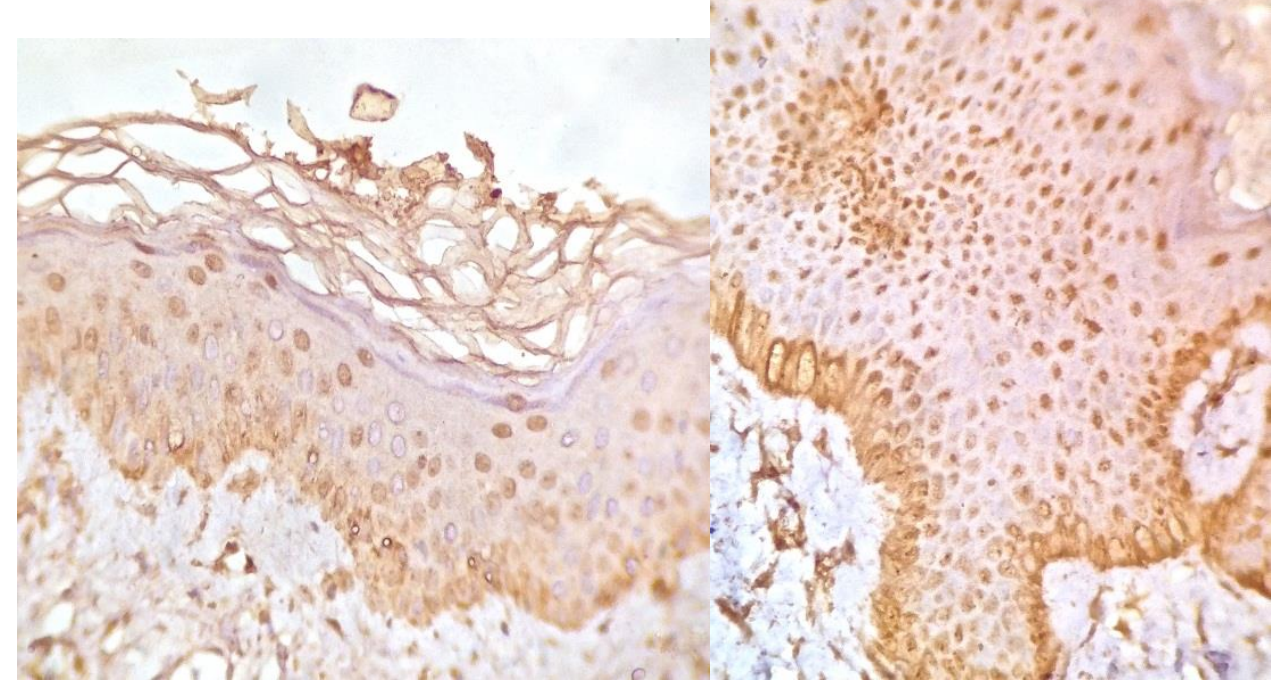

(a)

(b)

Fig(4): IHC staining of b-FGF of lesional skin before(a) and after(b) 3 months of melanocyte transfer by sandpaper technique(b-FGF staining $\times \mathbf{4 0 0})$.

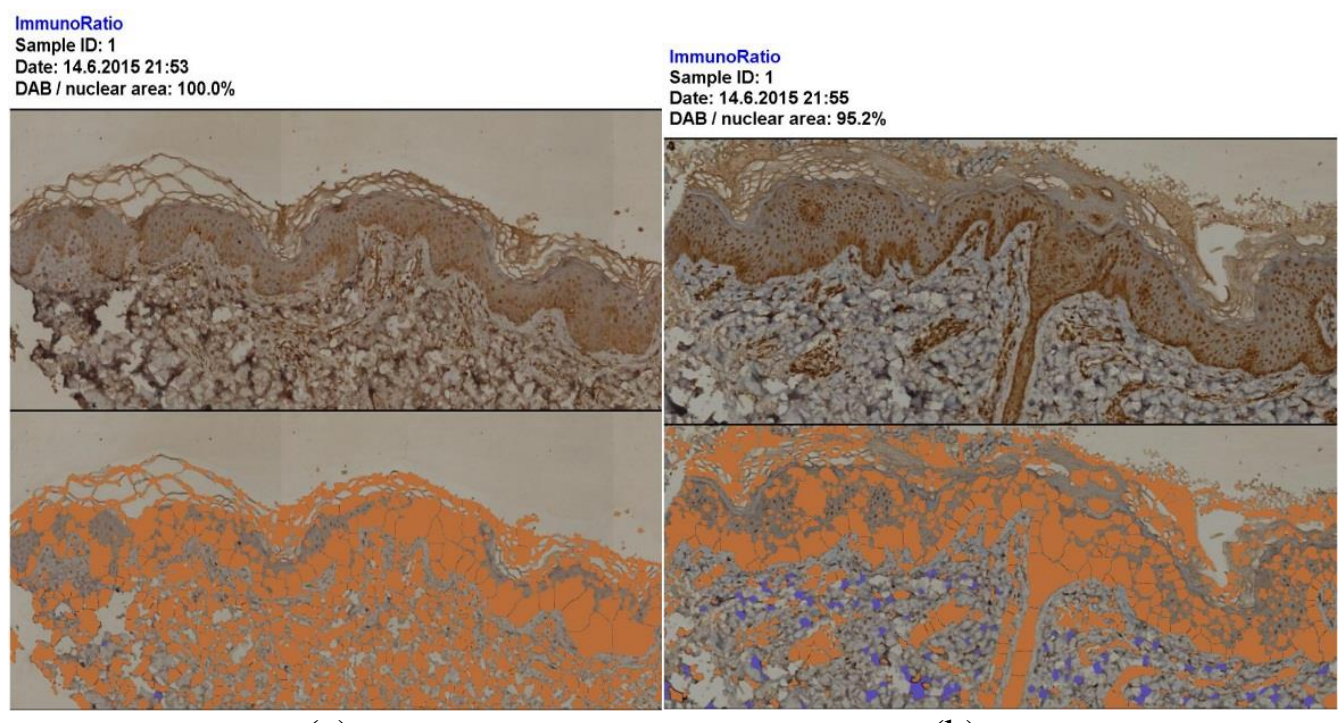

(a)

(b)

Fig(5): Image analyser of b-FGF staining of lesional skin before(a) and after(b) 3 months of melanocyte transfer by sandpaper.

DISCUSSION

This study is mainly focused on determining the change in TNF $\alpha$ and $b-F G F$ in lesional and perilesional skin in vitiligo patients before and after melanocyte transfer by sandpaper technique as one of the surgical therapeutic option of vitiligo..

Comparing the keratinocyte nuclear staining of b-FGF in lesional and perilesional skin with their staining in normal control there was a highly significant increase .This is consistent with the results of Moretti, et al, $2009^{[3]}$.

Regarding the keratinocyte nuclear staining of TNF $\alpha$ of lesional and perilesional skin,there were a significant increase and a highly significant increase respectively with its keratinocyte nuclear staining in the normal control.This can be explained by the fact that oxidative stress of keratinocytes is an early change in the pathogenesis of vitiligo with subsequent increase in TNF $\alpha$ and keratinocyte apoptosis together with the paracrine 
inhibition of melanocytes by $\mathrm{TNF}^{[4,8]}$. These results go with the observation of Silvia Moretti et al in their study[3]. They attributed the increased apoptotic keratinocytes in vitiligo skin biopsies confirmed by TUNEL assay and electron microscopy to increased TNF- $\alpha$ levels in the skin.

The clinical results of the melanocyte keratinocyte comixture transfer by sandpaper technique were as follow, $47.1 \%$ of patients showed mild improvement,35.3\% of patients showed good improvement,and $17.6 \%$ of patients showed no improvement.On the other hand, the results of Quezada N, et al,2011. ${ }^{[7]}$ showed mild improvement in $18 \%$ of patients,good improvement in $73 \%$ of patients ,and no improvement in $9 \%$ of patients. This may be explained by possible difference in Fitzpartic skin types in the patients of our study and their study or the difference in the number of patients included in the two studies.

At the end of these 3 months, skin biopsies were taken from the lesional and perilesional areas of all the patients .These biopsies were prepared to be evaluated by the same method mentioned before(i.e.subjective method).

The results of keratinocyte nuclear staining of $\mathrm{TNF} \alpha$ of lesional and perilesional skin showed a statistically highly significant increase after treatment with melanocyte transfer in comparison with their staining before treatment.

It was suggested that the prolonged sustained increase of TNF $\alpha$ may have a therapeutic role (at the molecular level) in vitiligo as a late event by induction of a coinhibitory signals between $\mathrm{T}$ helper1(Th1) cells and monocytes. This was attributed toTNFa induced up-regulation of the programmed cell death protein 1(PD-1) levels on activated (Th1) cells that can bind to the programmed death-ligand(PD-L)expressed on monocytes and induces IL-10 production by monocytes that inturn inhibit the activated Th1 cells with subsequent inhibition of $T$ cytotoxic cells and inhibition of cellular dependent autoimmunity and also cause immunomodulation by increase Th 2 over Th1 by the increased IL-10 ${ }^{[9]}$.
The comparison of keratinocyte nuclear staining of b-FGF in lesional and perilesional skin before and after treatment with melanocyte transfer showed a statistically highly significant increase after treatment in lesional skin and a statistically highly significant decrease after treatment in perilesional skin.

It is well known that b-FGF is a paracrine mitogen to melanocytes released from the neighbouring keratinocytes [3]. The statistically highly significant increase in keratinocyte nuclear staining of b-FGF in lesional skin after treatment with melanocytekeratinocyte comixture transfer can be explained by its production by the newly transmitted keratinocytes to lesional skin.The highly significant decrease in keratinocyte nuclear staining of $b$-FGF in perilesional skin after treatment can be explained by the expected decrease in the released b-FGF from the apoptotic keratinocytes in perilesional skin without new keratinocyte transfer to this perilesional skin.

Using Image analyser,there was a statistically significant increase of keratinocyte nuclear staining of $\mathrm{TNF} \alpha$ of lesional and perilesional skin after melanocyte transfer.These results go with the results of subjective methods and the explanation was discussed before.

By Image analyser,there was a statistically significant decrease in keratinocyte nuclear staining of b-FGF in lesional skin after treatment with melanocyte transfer and there was no significant change in perilesional skin before and after melanocyte transfer.

These results can be attributed to some properties of standardization of this method including,the absence of counterstain is maindatory of this method, the image analyser detect any degree of intensity of staining even if faint and it needs computerized cutting of the histopathological blocks at the same time.All these properties should be put in consideration before using this method to be standardized ${ }^{[10]}$.

Our patients biopsies were taken at different times before and after treatment, the blocks,also were not computerizly cut,and the counter stain was present .All these factors 
may play role in making this method not standardized for evaluation.

\section{REFERENCES}

1-Kyriakis KP, Palamaras I, Tsele E, Michailides C, and Terzoudi S: Case detection rates of vitiligo by gender and age. Int J Dermatol; 2009;48:328-9.

2-Alikhan A, Lesley M. Felsten, Meaghan Daly,and Vesna Petronic-Rosic:Vitiligo: A comprehensive overview. J Am Acad Dermatol; 2011;65:473-91.

3-Silvia Moretti, Paolo Fabbri, Gianna Baroni, Samantha Berti, Daniele Bani, Emilio Berti, Romina Nassini, Torello Lotti and Daniela Massi: Keratinocyte dysfunction in vitiligo epidermis: cytokine microenvironment and correlation to keratinocyte apoptosis, Histol Histopathol; 2009;24: 849-857.

4-Lee AY, Kim NH, Choi WI, Youm YH: Less keratinocytederived factors related to more keratinocyte apoptosis in depigmented than normally pigmented suction-blistered epidermis may cause passive melanocyte death in vitiligo. J Invest Dermatol; 2005;124: 976983.
5- Shi SR,Gu J,Kalar KL,et al:Antigen retrival technique:A noval approach to immunohistochemistry on routinely processed tissue sections.Cell Vision; 1995;2:6-9.

6-Aperio technologies Inc: IHC nuclear image analysis .User's Guide.Part Number Revision:MAN-0027,Revision B; 2007;7-40.

7-Quezada N, App C, Filho M, De La Sotta P, Uribe $P$ (2011): Melanocytes and Keratinocytes Transfer Using Sandpaper Technique Combined with Dermabrasion for Stable Vitiligo. Dermatol Surg; 37: 192-198.

8-Dell'Anna ML, Picardo M:A review and a new hypothesis for non immunological pathogenetic mechanisms in vitiligo. Pigment Cell Res; 2006; 19: 406-411.

9-Said EA, Dupuy FP, Trautmann L, Zhang Y, Shi Y,and El-Far M: "Programmed death-1induced interleukin-10 production by monocytes impairs CD4+ $\mathrm{T}$ cell activation during HIV infection". Nat. Med. 2010;16 (4): 452-9.

10-Rizzardi AE,Johnson AT, andVogel RI:Quantitative comparison of immunohistochemical staining measured by digital image analysis versus pathologist visual scoring.Diagnostic Pathology; 2012;7:42 